\title{
Sagittal balance spinopelvic impact on kyphoscoliosis thoracolumbar or lumbar and/or hypnosis: analysis o fase series of cases
}

\begin{abstract}
The proper restoration of the sagittal vertebral imbalance and the selection of the surgical technique in rigid deformities of the spine today are the necessary to achieve better clinical outcome pillar. To evaluate the overall global sagittal axis and correlate the spinopelvic parameters of patients who underwent surgery with kyphoscoliosis and/or kyphosis. A retrospective study of 16 cases from January 2011 to December 2014. In 7 (44\%) patients underwent spondylectomy and in $9(33 \%)$ pedicle subtraction. Preoperative Cobb angle of $79^{\circ}$ kyphoscoliosis, with an average percentage of correction in the coronal plane $59 \%$ and $57 \%$ in the sagittal plane of $32 \%$ was observed. Comparing the angular parameters showed improvement of the negative sagittal unbalance, with a plumb line $\mathrm{C} 7$ line of the plumb bob with a displacement of $12.6 \mathrm{~mm}$ posterior to the upper rear corner of S1. Global sagittal axis increased with a decreased in retroversion of the pelvis and increased knee flexion. The lumbar lordosis showed a loss of $12^{\circ}$ with correction and correspondence with the pelvic incidence of $37^{\circ}$. Complications in 11 patients, LCR fistula, nonunion, with blood loss of $700 \mathrm{ml}$, surgical time of 8 hours, and fusion $70 \%$ In patients with vertebral deformities and coronal plane is sagittal, the ideal realignment of the global sagittal axis and surgery play a key role in the preoperative period to avoid future complications.
\end{abstract}

Keywords: spinopelvic sagittal balance, kyphoscoliosis, kyphosis, vertebrectomy, pedicle subtraction
Volume 3 Issue 2 - 2019

\author{
Gomez N,' Krivoy J, ${ }^{2}$ Damas A, ${ }^{3}$ Avila A ${ }^{4}$ \\ 'Specialist in Neurosurgery, Hospital Ana Francisca Pérez de \\ León II, Brazil \\ ${ }^{2}$ Specialist in Neurosurgery, Head of the Neurosurgery \\ Department, Hospital Universitario de Caracas.UCV, Brazil \\ ${ }^{3}$ Specialist in Orthopedics and Spine Surgery, Chief of the Spine \\ Surgery Service, Hospital San Juan de Dios, Brazil \\ ${ }^{4}$ Specialist in Neurosurgery, Head of the Neurosurgery \\ Department, Hospital Universitario de Maracaibo, Brazil
}

Correspondence: Gomez Natascha, Specialist in Neurosurgery, Hospital Ana Francisca Pérez de León II, Brazil, Email nataschagomez2013@hotmail.com

Received: July 18, 2018 | Published: March 19, 2019

\section{Introduction}

Kyphotic deformities of the spine are defined as abnormal increases in curvatures in the sagittal plane in the dorsal direction of the spine. ${ }^{1}$ Congenital kyphosis or kyphoscoliosis is a posterior convex angular deformity of the spine associated with abnormalities. Kyphotic deformities of the spine are defined as abnormal increases in curvatures in the sagittal plane in the dorsal direction of the spine. Congenital kyphosis or kyphoscoliosis is a posterior convex angular deformity of the spine associated with abnormalities and a rotational deformity in the axial plane deformed during growth that may lead to severe kyphosis or kyphoscoliosis. ${ }^{2}$ The etiology of increased thoracic or thoracolumbar kyphosis in children and adolescents is Scheuerman's disease, congenital kyphosis and postural round back. Winter et al., they classified more than 15 major groups of kyphosis. Van Schirck et al. described three categories: type I, congenital vertebral body formation failure, which progressed more than 10 degrees per year in adolescence; type II, vertebral segmentation failure; and type III, which tend to progress rapidly and produce severe deformities of all types of kyphosis. ${ }^{4}$ However, the severity of the kyphotic deformity will depend on the length of the affected area and growth. The highest incidence is seen from T10 to L2. In congenital kyphoscoliosis, the complete lack of disc formation from the front to the back of the body is less likely to be curved by subsequent growth than in the case of complete failure of disc formation. The problem is further complicated in cases of segmentation defects that do not appear until advanced growth age: congenital kyphosis, which must be treated before it exceeds the normal limits of thoracic kyphosis. Likewise, the Scoliosis Research Society has accepted a normal margin of kyphosis $\left(30-40^{\circ}\right)$ measured between T3-T12; as long as its progression and any degree of kyphosis in the thoracolumbar or lumbar area are documented, it should be considered anomalous.
Von Rokitansky in 1844 describes the first article about a patient with congenital kyphosis. ${ }^{7}$ However, several studies have analyzed their natural evolution; Mc Master et al., ${ }^{8}$ followed 216 patients not treated for five years and found that many patients have back pain and significant functional disability due to spinal imbalance sagittal and coronal, associated with kyphoscoliosis or kyphoscoliosis, as well as cosmetic problems. In another study, Lenke et al., ${ }^{9}$ conclude that by using the posterior approach for vertebrectomy in 35 patients. The average rate was $51 \%$ correction in rigid kyphosis, observing less surgical time and loss of blood volume. Gavagaskar et al. ${ }^{10}$ in the year 2010, noted that the vertebrectomy, arthrodesis and fixation is a good alternative to the treatment of congenital scoliosis by default of segmentation, reporting that the total percentage of correction of the curve was $77 \%$, with $21 \%$ of neurological complications; as a result of compression direct and tension of the spine through of the deformed region that consisted in lower limb, paresthesias and painful phenomena, compared with Ponte and Smith's osteotomy Peterson, similar results to those reported in the literature. ${ }^{11}$ However, paraplegia can occur early, but it is more common during the adolescent growth spurt with a rapid increase in kyphosis. ${ }^{12}$ Surgical correction is the primary treatment for significant congenital spinal deformity. Your surgical correction may be associated with risks and significant challenges. The criteria for the indication of surgical treatment are: directly related neurological changes, kyphosis over 70 degrees, rapid curve progression, and pain that does not respond to conservative treatment. ${ }^{13}$

In the last few years, many surgeons have applied a variety of surgical techniques in the correction of congenital kyphosis, or kyphoscoliosis, already either by a single procedure rear, front or combined. The most of these studies focused on the in method innovation improvement of the corrective efficiency of the overall sagittal and coronal spinal 
balance, and reduction of the surgical complications. The process of surgical decision making is the selection procedure; between the Smith-Peterson osteotomy, pedicle subtraction osteotomy, and spine resection. The amount of correction needed to correct the deformity and evaluation of the spinopelvic parameters, determine the procedure and should be measured before the operation; however, it is still a problem that needs to be addressed..$^{14}$ Nevertheless, in patients with kyphosis and kyphoscoliosis, evaluation of the following spinal parameters should be considered: plumb line $\mathrm{C} 7$ that is in the range of 6-8 $\mathrm{cm}$ positive. The amount of correction provided by the pedicle subtraction osteotomy or vertebrectomy that is in the range of $9-10^{\circ}$ per level. A degree of correction is achieved per millimeter of resected bone. Also, in the patients requiring $10^{\circ}$ to $20^{\circ}$ of lordosis o $6-8 \mathrm{~cm}$ of $\mathrm{C} 7$ line correction plumbing, it is more appropriate to perform a limited number of objectives of the Programme that will be used as a basis for pedicle subtraction osteotomy, a less than the deformity fixes this previously merged. ${ }^{15}$ Bradford et al., ${ }^{16}$ first describe VCR spine resection, which provides the greatest amount of correction and is indicated in severe rigid deformities of the spine, such as congenital kyphosis, rigid multiplanar deformities, acute kyphotic deformities, a difficult procedure involving complete resection of the elements and the vertebral body including adjacent discs of one or more levels; providing controlled manipulation of the anterior and posterior regions simultaneously.

In a series of 35 children, Lenke ${ }^{17}$ observed significant improvements in the curve of up to $51 \%$ in the cases of Scoliosis, $55 \%$ of global kyphosis cases, $58 \%$ of angular kyphosis cases, $54 \%$ of kyphoscoliosis cases, and $60 \%$ of congenital scoliosis cases. A preoccupation important with CRVP is the possibility of neurological complications, which may result from direct neurological injury during bone resection or correction of the deformity. Lafage et al., ${ }^{18}$ reported in nine patients, sagittal plane deformities, primarily decreased thoracic kyphosis and increased lumbar lordosis in the in the progressive form of this disease, concluding that the analysis of the sagittal balance, the study of the spinopelvic parameters and the spinal fusion should be carried out very early. Changes in the sagittal plane of the trunk may be responsible for an imbalance of the sagittal trunk, severely affecting its correction and fusion. The ideal global vertebral realignment should aim to obtain at least one postoperative $\mathrm{C} 7$ plumb line with a displacement of less than $50 \mathrm{~mm}$, a postoperative PT of less than $25^{\circ}$ and an LL-PI in the range of $+/-9$, although with on this last point, other authors argue that an LL postoperative $\leq^{\circ}$-PI may predict clinical benefits. ${ }^{19,20}$ The acquisition of the bipedal posture meant for man the liberation of the upper limbs and brought about the modifications in the field of spinal statics and especially in the sagittal plane. Spinopelvic balance in the sagittal plane has been described as a line which connects the head to the pelvis and allows the assessment of sagittal balance, which is necessary to assess the shape of the pelvis in preoperative and follow-up of patients with deformities. ${ }^{21}$

Studies have confirmed that the sagittal balance is determined basically by the $\mathrm{C} 7$ line of the plummet. By convention, the balance positive sagittal is produced when the $\mathrm{C} 7$ line of plummet falls ahead of the upper rear corner of the plate terminal S1 and the sagittal equilibrium negative occurs when the $\mathrm{C} 7$ plummet falls behind this point. The average of the $\mathrm{C} 7$ plummet line with a displacement from the corner $\mathrm{S} 1$, the upper rear end of $\mathrm{S} 1$, has been found normal of $0.5 \mathrm{~cm}$. A displacement $>2.5 \mathrm{~cm}$ anterior or is considered to be abnormal. ${ }^{22}$ Recent studies have shown that sagittal balance should be the most important radiographic and reliable predictor of the clinical health status of patients who have spinal deformities. The positive sagittal balance is the radiographic parameter that is most highly correlated with the adverse result of health status. ${ }^{23,24}$ Some structural features of the pelvis in large part the measurement determine lumbar lordosis, as well as sagittal pelvic alignment and spinal balance. Different components, such as thoracic kyphosis (TK), LL, and pelvis, help define overall sagittal balance. Traditional knowledge explains that kyphosis is measured from the plate upper terminal from $\mathrm{T} 4$ to the plate lower terminal of $\mathrm{T} 12$ ranging from $10^{\circ}$ a $40^{\circ}$. Lordosis is measured from the upper end of the plate from $\mathrm{L} 1$ to the $\mathrm{S} 1$ end plate usually go from $40^{\circ}$ to $60^{\circ} .{ }^{25}$ There is an important chain of interdependence between the parameters of the pelvis and the spine. The influence of the pelvis on the sagittal spinal alignment has been described, among others, by Duval-Beaupère, who introduces the incidence of the pelvis (PI), the inclination of the pelvis (PT), and the slope of the sacrum (SS), which are angles to describe the shape and orientation of the pelvis. ${ }^{26}$

The PI is a non-positional anatomical parameter defined as the angle between the line perpendicular to the sacral plate at its midpoint and the line connecting this point to the axis of rotation of the femoral head. The PI angle determines the relative position of the sacral plate relative to the femoral head and determines the remaining variable parameters, which is equal to the sum of SS + PT. The SS is a positional parameter defined as the angle between the top end plate of $\mathrm{S} 1$ and a horizontal line extending from the anterior- inferior corner of the end plate S1. The degree of the sacral slope determines the position of the lumbar spine. ${ }^{27,28}$ The PT is a positional parameter, defined as the angle between the line connecting the mid-point of the upper sacral end plate to that of the femoral axis of rotation and the line extending vertically from the femoral axis of rotation. The spatial orientation of the pelvis, which varies according to position, is indicated with a greater or lesser degree of inclination forward (pelvic anteversion) or backward (pelvic retroversion) in relation to a transverse axis passing through the two femoral heads. The greater the angle of the pelvic tilt, farther away from the center of gravity is projected back of the femoral head. ${ }^{29}$ The correlation between the radiographic parameters and a pain perception and disability are tools that are fine documented. ${ }^{30}$ When the spinal column is in kyphosis or hyperextension, the compensating mechanisms are not enough to keep the plummet line C7 behind the femoral head; the only solution to keeping the line of gravity between the two feet is to bend the knees. Therefore, in the most severe cases, with unbalanced columns, patients may present with different signs of decompensation such as: forward bent trunk, retroversion of the pelvis, hip extension and knee flexion. Once a deformity of the column overcomes these compensatory mechanisms, surgical intervention is often requested. Surgery is the main means of compensatory mechanisms often requested. ${ }^{31}$

Surgery is the mainstay of treatment for patients with sagittal deformity. The indications include non-surgical treatment failure, curve progression, back pain, root symptoms and significant cosmetic deformity. The goals of surgery are to achieve a solid fusion with a balanced spine in both the sagittal and coronal planes, relieve pain and prevent progression. ${ }^{32}$ Several studies have shown that restoration of adequate alignment in the sagittal plane is necessary to improve significantly the clinical outcome and avoid subsequent pseudoarthrosis. These relationships have been documented in patients with spinal disorders, observing the fixed sagittal imbalance (FSI), also known as the syndrome "flat back" or kyphotic decompensation, which occurs in patients who have lost normal lumbar curvature or lordosis, 
or in the postoperative period of rigid kyphosis due to vertebrectomy, resulting in a positive balance for sagittal tilt of the trunk, anterior displacement of the center of gravity, and difficulty in maintaining a standing position, including hyperextension of the cervical and thoracic segment, with retroversion of the knees and hips. ${ }^{33,34}$

The flexibility of the spinal column should be evaluated clinically and radiologically standing, coronal and/or sagittal deformity of patients may decrease in supine or prone position due to moving segments. Antero-posterior foot and lateral radiographs of flexion, in supine position, of lateral support point and of flexion and extension, with lateral radiographs that can demonstrate the flexibility of the deformity. Consideration of spinopelvic parameters is fundamental in surgical planning. ${ }^{35}$ Fixed deformities can be managed by anterioronly, anterior and posterior deformities, are combined and posterior with recent advances in instrumentation and subsequent techniques only approaches, became very popular in recent years. Numerous studies have been published that guarantee the safety and efficacy of a posterior approach only for the treatment of all spinal deformities. ${ }^{36}$ Several studies have been conducted in the normal population and in patients with neuromuscular, degenerative, and spondylolisthesis, where they are observed changes in pelvic parameters and spinal cord of the sagittal balance of the spine. However, few studies have addressed the disorders of the spinal column in patients with kyphosis or kyphoscoliosis. ${ }^{37,38} \quad$ Subsequent approaches of only one approach include: Smith-Peterson's osteotomy, pedicle subtraction osteotomy, and spine resection. The amount of correction needed to correct the deformity determines the surgical procedure, and must be measured before the operation. ${ }^{39}$

\section{Objective}

To evaluate the sagittal equilibrium of the rachis through of the clinical and radiological evolution of patients with kyphoscoliosis and/or kyphosis who underwent a Pedicle Subtraction Osteotomy and Vertebrectomy and correlate the intra- and post-operative spinal and pelvic parameters in the Spine Surgery and Orthopedic Service.

\section{Specific objectives}

I. Analyze the demographic descriptive variables (age, sex, symptoms and associated pathologies).

II. Evaluate by imaging studies (Spine X-ray panoramic anther posterior, Lateral and Lateral inclinations, Panoramic AnteroPosterior and Lateral Tac from C1 up to femoral and MRI of the thoracolumbar spine): Type of kyphoscoliosis and/or kyphosis according to Wenger classification, Cobb angle (intra-postoperative), wedging angle, degree of correction and vertex, plumb line $\mathrm{C} 7$, vertical sagittal axis line (VAS), pelvic incidence (PI), T9 sagittal line, pelvic tilt (PT), sacral slope (SS), kyphosis thoracic, lumbar lordosis.

III. Assess coronal imbalance: the antero-posterior imbalance (displacement in $\mathrm{mm}$ from the spinous process of $\mathrm{T} 1$ to a perpendicular line traced from the center of the sacrum) and sagittal unbalance.

IV. Evaluate the overall sagittal axis GSA: it is defined as the angle formed by a line from the middle point of the condyles femoral in the center of $\mathrm{C}-7$, and a line from the midpoint between the femoral condyles to the upper posterior corner of the terminal plate S-1 sacral.

V. Determine the correlation of the angular parameters intra and post-operative spinal pelvic exams after surgery PSO and VCR and their influence on the GSA.

VI. Analyze the neurological evolution and rate of pseudoarthrosis at 6 months postoperative, surgical time, bleeding, surgical technique used and complications.

\section{Methods}

\section{Type of study}

The present study was a retrospective and descriptive.

\section{Population and sample}

The population studied was made up of the total number of patients who were operated on surgically with Vertebrectomy, Spinal resection in wedge and Pedicle Subtraction Osteotomy in the Orthopaedic and Surgery Service of Spine of the San Juan de Dios Hospital, between January 2011-December 2014; with the diagnosis of kyphoscoliosis and/or kyphosis determined by clinical and imaging studies ( $\mathrm{Rx}$ of Panoramic column in projections antero-posterior, lateral- Tac of Column ) and MRI, taken in its entirety as a sample, of which 16 patients were selected. The following are considered as criteria for inclusion: patients with kyphoscoliosis congenital and/or thoracolumbar kyphosis or lumbar spine of both sexes, age between 3 and 18 years old, no history of surgery for previous spine, no abnormality radiographic (isthmic lysis before or after during the study, spondylolisthesis) with kyphosis curves $>70^{\circ}$, in the Lateral tilt projections and anteroposterior standing. Patients with pathology of deformities Congenital: Kyphoscoliosis and Kyphosis of the Spinal Column of management surgical in the institution during the period January 2011-December 2014. Presence of neurological involvement clinic. Patients with surgical indication of Vertebrectomy and/or Pedicle Subtraction through posterior approach. Patients over 18 years of age were excluded, as well as patients for medical management with orthosis and physiotherapy, patients undergoing previous spinal surgery, and disc disease of infectious origin intraspinal, tumoral and inflammatory abnormalities and cases of idiopathic, neuromuscular scoliosis.

\section{Procedure}

Age, sex, an etiology were analyzed, as well as the clinical features, unbalance and radiographic measurements of the spinopelvic parameters, surgical time, blood loss. All the medical records of the Medical Histories Department of San Juan Hospital were reviewed in patients with congenital kyphoscoliosis and kyphosis, applying the data collection instrument to them, verifying which of them fulfills the criteria of the inclusion and exclusion established for the purpose of obtaining the sample. This work was reviewed and approved by the hospital's ethics committee. The radiological analysis was carried out by panoramic radiographs in antero-posterior, lateral, and lateral projections, respectively. Lateral inclination of the foot and panoramic spine from the inner ear canal to the femoral heads for each patient. As complementary tests for the diagnosis of these alterations, a panoramic spine CT scan from $\mathrm{C} 1$ to femoral condyles was requested, among others. Intrathoracic pathology was ruled out in the left thoracic curves using NMR. In each lateral $x$-ray, the following pelvic parameters were evaluated: The VAS (vertical sagittal axis), measures the distance between the plummet line and the posterior corner of the plate of S1. Sagittal equilibrium is basically determined by the plummet C7 line. The half plummet C7 displacement from the upper 
rear corner of S1 has been found to be $0.5 \mathrm{~cm}$. A displacement $>2.5 \mathrm{~cm}$ before or after is considered abnormal the plummet line of $\mathrm{C} 7$ is a line that from $\mathrm{C} 7$ a vertical line between the center of the body of $\mathrm{C} 7$ and the lower back angle of the body of L5 VN $(-3 \mathrm{~cm})$, falls in the front or behind the femoral heads. Different components, such as kyphosis thoracic (TK), the LL and pelvis help to define the overall sagittal balance. The lumbar lordosis is the measured parameter between L1 upper plate and plate of L5 (VN 43 to 61) and kyphosis thoracic TK angle between saucer of T4 and the lower of T12 (VN 41 a 68 . The sagittal line in T9 (LS-79) on angle is formed by the vertical that passes through the CCF and a line that joins the CCF with the T9 center. The mean value is 11 degrees. The vertical of CAE and the axis global sagittal (GSA) allows a study of the more complete of the rigidity of the deformity of the spinal column and the pelvis.

There is an important chain of the interdependence between the balance sagittal, pelvis and spine, angles to describe the shape and pelvic orientation. The Pelvic tilt (PT) that is corresponds to the angle between the line that connects the midpoint of the plate of the sacrum to the bicuspid femoral axis and the plane vertical (VN 12 to 18). LL is closely related to the orientation of the pelvis as directed is determined by the angle of PI. The Sacred Slope (SS) is a parameter of position is defined as the angle between the upper end plate of $\mathrm{S} 1$ and a horizontal line extending from the front lower corner of the plate terminal S1. The grade of the slope sacral determines the position of the lumbar spine, from the sacral platform forms the basis of the column and the (PT) is a parameter of position that is measured as the angle between the line connecting the point sacral end plate medium greater than that of the femoral axis of rotation and the line that extends vertically from the femoral rotational axis and indicates the spatial orientation of the pelvis; the greater the angle of the pelvic tilt, farther away from the center of gravity is projected back of the femoral head. The surgical correction is performed by osteotomy of pedicle subtraction (PSO): that consists of an osteotomy in the form of a transpedicular wedge at the back, resection of the spine (VCR): which is a method of correcting the severe coronal deformity and resection circumferential wedge-shaped vertebrae with the use of translation technique - Freehand rotation with image intensifier rearend instrumentation (Pedicle Screws) with amplifier of images.

For the collection of data, it is necessary to use a tab: age, sex, a etiology clinical features, angle Cobb's kyphosis, wedging preoperative and post-operative, correction percentages, apex of the kyphosis or kyphoscoliosis, abnormalities intraspinal, unbalance, picture according to the neurological scale of Frankel in pre-op and post-op operative, surgical time, loss blood, complications and fusion. After the data tabulation is completed, the proceeded to the analysis of the results using: frequency and value percentage. From the foregoing, the interpreted the results and were established conclusions and recommendations in order to stimulate academic and scientific development. Once the information was collected, the data were analyzed in three steps. First, we carried out a descriptive study of the demographic and morphological parameters of the cohort; secondly, we calculated the frequency and percentage value of the nominal variables (unbalance and angular parameters) and thirdly, the reciprocal variations and correlations of all parameters in both groups were studied in agreement with each other by means of the chisquare Person test. In all the contrasts the differences were considered significant if the $\mathrm{P}$ was $<0.05$. For statistical analysis, the following are used designed a database using the statistical programmer JMPSAS 11.0, and in the case of the diagnostic values, the used the EPIDAT 3.0 application.

\section{Results}

From January 2011 until December 2014, 16 patients with Congenital Kyphoscoliosis and Kyphosis thoracolumbar and/or lumbar underwent he surgical correction in our hospital. It was obtained for the description of the sample, tables with frequency and value percentage of patients by age, sex, etiology and type of associated pathology. In the sample distribution population by age group and sex; in relation to sex, predominance was observed in the of males $10(62 \%)$ over females $6(38 \%)$, as for the distribution by age were treated with this technique patients between 1 and 18 years, with the predominant group being aged between $10-18$ years $(56 \%)$ of cases. All the patients were evaluated by medical records and X-rays in projections antero-posterior and lateral, and inclination lateral (Bending test) of the rachis complete preoperative and post-operative, as well as the use of the Panoramic Tac from C1 to heads femoral, evaluating different radiological spinopelvic parameters, ruling out intrathecal pathology by MRI. In regard to etiology, it was observed that 7 (44\%) patients presented congenital deformities: kyphoscoliosis by default of formation, $3(19 \%)$ cases of wedge vertebrae and $6(38 \%)$ cases of kyphosis according to Wenger classification: $4(25 \%)$ patients had type 1 (thoracic form) and $2(13 \%)$ presented type 2 (thoracolumbar form). Of all the patients, when evaluating the location of the apex of kyphoscoliosis and/or kyphosis, the majority of 5 cases had the vertex of kyphosis in the thoracolumbar or lumbar segment (T12-L2/ L2-L4), 3 cases in the lower thoracic spine (T11-T12), 6 cases in the middle thoracic segment (T5-T9) and only 2 cases had the vertex in the upper thoracic spine (T1-T4). In this series, it was found that the $2(13 \%)$, the highest percentage was cases with spinal dysraphism lumbosacral, while only in 3(19\%) patients observed marrow Anchor and/or diastematomyelia; respectively, and the rest of the patients no anomalies $11(68 \%)$. The average Cobb angle of the kyphoscoliosis with the coronal curve in the preoperative was $79^{\circ}$ (range $70^{\circ}$ to $\left.110^{\circ}\right)$ and the average angle in the immediate postoperative period was $32^{\circ}$ (range 14 to $39^{\circ}$ ), with a correction rate of $59 \%$ and in the sagittal period was $57 \%$, with an initial average angle of $85^{\circ}$ (range $78-105^{\circ}$ ), and an average angle in the postoperative period was $27^{\circ}$, with the technique of vertebrectomy and resection circumferential of the wedge-shaped vertebrae. All the patients had curves secondary cephalic and caudal.

In kyphosis-like deformity, the average preoperative angle was $75^{\circ}$ (range 72 to $80^{\circ}$ ), and the angle average postoperative period was $42^{\circ}$, (range 37 to $60^{\circ}$ ), which means that corresponds to a correction rate of $33 \%(25-41)$ with the use of a stand and pedicle subtraction with improvement of sagittal imbalance $(11 \mathrm{~cm})$ and correction of the coronal plane $(0.48 \mathrm{~cm})$. By observing the parameters Spinopelvic angles between patients with kyphoscoliosis and kyphosis, it was obtained on average that the plummet from $\mathrm{C} 7$ was $12.6 \mathrm{~mm}$ posterior to the corner upper back of $\mathrm{S} 1$ on average (range -88 to $60 \mathrm{~mm}$ ): unbalance sagittal negative was evidenced in 10 cases, with $(47 \mathrm{~mm})$ displacement of the $\mathrm{C} 7$ plummet. The balance sheet in the sagittal plane is well maintained in the 2 cases and found: imbalance sagittal positive in 4 cases, with $(35 \mathrm{~mm})$ of anterior displacement of the $\mathrm{C} 7$ plummet on average with an increased thoracic kyphosis (T2-T11), significant difference and displacement from the trunk forward, which is positive, without compensation from the lordosis. In the last few years of tracking, the plummet line from $\mathrm{C} 7$ was $1.5 \mathrm{~mm}$ posterior to the corner upper back of $\mathrm{S} 1$ on average range (-36 to $38 \mathrm{~mm})$. The patients with negative sagittal imbalance is reduced to 5 cases, with $(27 \mathrm{~mm})$ of rearward displacement of the plummet $\mathrm{C} 7$ on average, 
and improved significantly compared to before the operation. The analysis of the (axis global sagittal) GSA increased by a decrease in the retroversion of the pelvis $(-0.83)$ and the increase in flexion from the knee (0.28). The sagittal displacement of T2-T11 has a strong relationship with the pelvic incidence and tilt pelvic floor. No compensation was observed between thoracic kyphosis and lordosis lumbar, with displacement of the line sagittal of $\mathrm{T} 1$ and $\mathrm{T} 9$, which was related to pelvic tilt and the sacred slope.

Likewise, it was reported that reciprocal correlations between the angular parameters in patients with kyphosis and kyphoscoliosis, not shown alteration of lumbar lordosis (L1-L5) and L1-S1) and the slope of the sacrum. It was evidence that the pelvic incidence was related to thoracic kyphosis, the lordosis (L1S1), the sacral slope e pelvic tilt, but not with the constant sagittal displacement of T1. Lumbar lordosis showed a loss very significant of 12.5 degrees from the prone position intraoperative to standing position postoperative, with a correction and correspondence between the preoperative middle pelvis, the 37 -degree pelvic incidence, and the $25^{\circ}$ sacral slope while the postoperative mismatch measured 3.2 degrees. The fusion of the thoracolumbar spine preserves the global sagittal axis better than the correction of the thoracic fusion. The approach used was later in 7 (44\%) patients' congenital kyphoscoliosis by defect of formation, the technique of vertebrectomy and vertebral resection in wedge, arthrodesis and fusion and in $9(33 \%)$ cases of kyphosis, the technique of pedicle subtraction, arthrodesis and posterior fixation was used. The Ponte osteotomy was performed in two segments in 9 (33\%) cases and allowed to improve the correction of the deformity and to increase the flexibility of the curve. The average intraoperative blood loss was $700 \mathrm{ml}$ (500-2500), which was determined that patients required transfusion of concentrated blood cells red, in all patients was used postoperative drainage, with an average bleeding during the postoperative period of $200 \mathrm{cc}$. As for the assessment of postoperative fusion, good fusion was observed in $(70 \%)$ cases and in $6(37 \%)$ cases, pseudoarthrosis with material fatigue and protrusion of the pedicle rod and screw. It was found that the average time of surgery varies from 7 to 8 hours and the time hospitalization was 5 days $(70 \%)$. The most frequent postoperative complications were: 2 incidental urinary ruptures repaired in the same surgical procedure, 2 cases of CSF fistula, 2 surgical wound infections with pressure ulcer and the need for wound debridement and healing, 2 patients presented functional segmental kyphosis proximal to the level of the instrumentation defect and 2 cases of pseudoarthrosis due to lack of bone graft fusion at the osteotomy site and 1 patient with intraoperative transitory paralysis due to ischemia of the spinal cord. Some complications were transient with an average total improvement of 8 weeks. In the clinical neurological evolution, 12 patients were classified as Frankel E and kept this scale in the postoperative period. In four patients with kyphoscoliosis, the following was observed in the evolution Franke 1A, evolving satisfactorily in the early neurological period postoperative period. Patient follow-up was between 6 months and 2 years, and it was noted that $70 \%$ of the patients received physical therapy postoperative period and $90 \%$ had improvement in pain. In no patient is there observed complications neurological.

\section{Discussion}

The surgical goal of congenital kyphosis or kyphoscoliosis is to decompression of the neurological elements and the correction of the deformity. Circumferential decompression is recommended for to eliminate the apical region of the deformity that involves the tail spinal horse, followed by instrumentation of a vertebra by and another one below the non-segmented sector, so that with the a trapezoidal vertebra develops on either side of the ends of the bar, thus compensating for at least part of the kyphosis. In case of severe kyphosis, the resection of the region apical deformity is performed is often performed by the pedicle subtraction osteotomy (PSO), vertebrectomy (VCR) and wedge resection procedures. Considering that in a mild deformity, the Smith Peterson osteotomy procedure may be sufficient to achieve satisfactory results. In the current series, the patients were divided into two groups of according to the severity of the kyphosis and the characteristics of the shape of the apical segment, and the corresponding procedures. In both the $7(44 \%)$ patients with congenital kyphoscoliosis by default of training, which was carried out using the technique of vertebrectomy and vertebral resection in wedge, arthrodesis and fusion and in the group of $9(33 \%)$ cases of kyphosis and vertebrae in wedge, in which the technique of pedicle subtraction, arthrodesis and rear fixation, the following were achieved good radiological results, correction of the global sagittal axis, with reduction of sagittal imbalance negative and clinical improvement after the surgery. ${ }^{21}$ The sagittal balance of the vertebral column and the parameters spinopelvic play an important role in clinical evaluation, surgical planning and postoperative outcomes.

Booth et al., ${ }^{16}$ in corrective surgery for deformities observed that the surgical results were significantly related to with the improvement of sagittal imbalance. Patients who had poor sagittal contour correction were less satisfied with their surgical results. Although the pedicle subtraction osteotomy is more technically demanding and more susceptible to complications than the Ponte Osteotomy; the PSO provides the advantage of obtaining correction through the three columns, while the posterior and the intermediate columns are shortened, this osteotomy does not lengthen the anterior column by avoiding stretching of the main vessels and anterior viscera to the spine..$^{32}$

An average of $30^{\circ}$ to $40^{\circ}$ of correction can be achieved with a single level of PSO..$^{35}$ Ideal candidates for an PSO are patients with a fixed sagittal imbalance of more than $12 \mathrm{~cm}$ and those patients with a circumferential arthrodesis along the length of multiple segments. ${ }^{37}$ Lenke et al., ${ }^{5}$ reported its results in a series of 35 patients with OSP; significant regional radiological changes with improved sagittal and coronal imbalance, between the second postoperative year and final follow-up, with only $10(29 \%)$ cases of pseudoarthrosis. Patient satisfaction (87\%) and function $(69 \%)$ were high after more than 5 years of follow-up. Spine resection (CRS) was introduced by Suk et al., ${ }^{14}$ and popularized in recent years by Lenke ${ }^{17}$ for severe rigid deformities of the spine despite the fact that it is a technically demanding procedure with a high rate of complications neurological; it allows several advantages: as well as manipulation in every direction under simultaneous control of both sites, front and back of the column and provides a better and more effective correction, with time reduction total operation and the amount of blood loss, avoiding opening of the thoracic cavity and pleura. Lewis et al., ${ }^{30}$ reported an average correction rate of $62 \%$ in the coronal plane and $72 \%$ in the plane sagittal in its series of 102 patients with severe kyphosis.

This data correlates with those obtained in this study, with a preoperative Cobb angle. of kyphoscoliosis $79^{\circ}$ and postoperative of $32^{\circ}$, obtaining a percentage average curvature correction of $59 \%$ in the coronal plane and $57 \%$ on the sagittal plane by means of the vertebrectomy and resection technique of wedge-shaped vertebrae. In 
relation to the kyphosis-like deformity, the angle preoperative average was $75^{\circ}$ (range 72 to $80^{\circ}$ ), and the angle average postoperative period was $42^{\circ}$, (range 37 to $60^{\circ}$ ), which means that is a correction rate of $33 \%$, with the use of Ponte's Osteotomy and Pedicle Subtraction with improvement of the sagittal imbalance and correction of the coronal plane and $57 \%$ on the sagittal plane using the vertebrectomy technique and wedge resection of vertebrae. In relation to kyphosis deformity, the average preoperative angle was $75^{\circ}$ (range 72 to $80^{\circ}$ ), and the average postoperative angle was $42^{\circ}$ (range 37 to $60^{\circ}$ ), which corresponds to a correction rate of $33 \%$, with the use of Ponte Osteotomy and pedicle subtraction with improvement of sagittal imbalance and correction of the coronal plane. In the same way, a predominance of the congenital defect was observed in $7(44 \%)$ patients due to failure of formation, followed by $3(19 \%)$ cases with wedge vertebrae in kyphoscoliosis; and 6 (38\%) of kyphosis located mainly in the thoracic and thoracolumbar region, which are of worse prognosis as to the progression of the curve; in accordance with the with the literature; therefore, the results obtained in terms of correction of the main curve are satisfactory, and it is inferred that the Longterm follow-up will allow us to determine the correction of sagittal balance of deformities and results; as described by Lenke et al., ${ }^{9}$ with the use of vertebrectomy in severe rigid kyphosis. ${ }^{25}$ So, as far as it can be inferred that the above-mentioned some authors regarding the importance of the type of defect congenital, location and evaluation of the overall sagittal balance constitute a progression prediction factor and postoperative clinical results.

Patients must be evaluated for intra-spinal abnormalities, about $20-40 \%$ of deformities congenital spines are associated with these abnormalities If the patient has neurological disorders such as the spinal malformation divide, myelomeningocele or anchored cord, in correction of the deformity significant care must be taken to avoid the possible compromise of the neurological function. ${ }^{34}$ Prahinski et al., ${ }^{33}$ suggests that in these congenital deformities associated with intraspinal pathologies, should first be treated by initial surgical treatment of the intramedullary pathology, and secondary surgical treatment of the deformity in a separate stage 1-2 weeks or 1-2 months later. In relation to the associated pathologies, 2 were observed (13\%) cases with spinal dysraphism lumbosacral, while only in 3(19\%) patients were observed cord Anchor and/or diastematomyelia; respectively, with a decision prior to surgical correction of the deformity, which is consistent with the reported in the literature, and the rest of the patients without anomalies $11(68 \%)$. Regarding the neurological evolution of control in the immediate postoperative period according to the Frankel scale, type of technique and approach used, as well as surgical time; in our studio, we confirm preference for use of the exclusive posterior approach, in 7(44\%) patients with kyphoscoliosis, with vertebrectomy and resection technique wedge-shaped vertebral column , arthrodesis and fusion; and in $9(33 \%)$ with kyphosis, who underwent pedicle subtraction osteotomy and Ponte Osteotomy. since it allows a good stabilization and correction of the deformity, reporting less surgical time, obtaining good clinical and neurological evolution in the patients with kyphoscoliosis, progressing satisfactorily in the postoperative period, results similar to those of the literature.

Numerous studies have been published that guarantee the safety and efficacy of using the posterior approach alone for the treatment of all spinal deformities. We believe that careful manipulation and continuous monitoring of spinal cord function are crucial to maintaining intact neurological. ${ }^{36,37}$ Likewise, a preoccupation important with vertebrectomy and wedge-shaped vertebral resection, it's the potential for complications neurological, which may result from a direct neurological injury during the bone resection or correction of the deformity reported that an overall rate of $34.3 \%$ of complications and a $17.1 \%$ rate of neurological complications. The patients from our series presented within the early complications in 6 (33\%) cases of pseudoarthrosis, 2 patients with LCR fistula, 2 cases of surgical wound infection, and dural dehiscence with correction of the without neurological alterations. functional and with a fusion $(70 \%) .{ }^{32}$ In relation to the evaluation of global sagittal balance and spinal parameters in patients with congenital kyphosis or kyphoscoliosis, some patients maintain a normal sagittal spinal balance with a compensatory corresponding to lumbar hyperlordosis. Unfortunately, in our study, 10 of the cases were found to have a negative sagittal imbalance, which is different from ankylosing spondylitis or Scheuerman's disease. The formation of negative sagittal imbalance is due to secondary hyperlordosis and thoracic hypokyphosis due to focal kyphosis. After the success of the surgery, along with the correction of kyphosis, it was also adjusted for compensatory hyperlordosis and thoracic hypokyphosis, which can improve the overall balance of the spine. If kyphosis cannot be compensated for by the change in the curvature of the lumbar and thoracic spine, the patient will have a forward tendency of the trunk and consequently have a positive sagittal imbalance, which can be directly improved for corrective surgery.

In our series, most of the people patients with sagittal imbalance negative or positive had a good balance after the corrective surgery. The patients showed improvement in 5 cases of the negative sagittal imbalance, with no forward trend of the trunk next to the deformity of the local kyphosis and only in 2 cases the balance sagittal remained positive, along with the improvement of the sagittal balance, and relief of the pain. In congenital kyphosis, or kyphoscoliosis, vertebral deformity has already existed since birth, which deteriorates after a growth. In some patients, the neurological element can be adapted to the commitment gradually during the long-term development of the deformation and the functions neurological can be maintained integrals. ${ }^{9}$ In our series, less than $50 \%$ of the cases had different degrees of neurological symptoms, and most of them had vertex kyphosis in the thoracic and thoracolumbar spine. Our results indicate that surgical correction of kyphoscoliosis and kyphosis, using the selection of surgical techniques and a thorough analysis of the stiffness of the deformity, the spine vertebral and pelvic (PI, PT and SS) parameters, and the mechanisms are of summation importance in order to be able to offer a solution adapted to these needs patients, resulting in the avoidance of possible complications, lower surgery times, days of hospitalization and bleeding intraoperative procedures are acceptable. However, each one of these procedures carries certain risks and they're technically demanding, which is why that preoperative optimization as well as the planning The procedure will allow a satisfactory angular correction to be obtained, achieving an ideal realignment of the global sagittal vertebral axis, even with partial improvement of the spinal parameters, which is very likely to translate into clinical benefits; although with respect to the latter, it is very likely that last point other authors argue that it can be accurately predicted by studying the sagittal axis of the spine, the clinical success after surgery. These patients should be followed up and further research after the end of growth in relation to spinal compensation.

\section{Conclusion}

I. Congenital kyphoscoliosis and thoracic or thoracolumbar kyphosis are confirmed to be predominantly in males between the ages of 10 and 18, with the pathology being an important 
factor in the Physiopathology and Surgical Correction not this diseases published by other authors.

II. The evaluation of sagittal equilibrium is the radiographic and reliable predictor of the clinical health status of patients with spinal deformity.

III. It shows that the correlation of spinal parameters and evaluation of the global sagittal axis and coronal imbalance are fundamental in preoperative planning and in the selection of the surgical technique to achieve a balanced spinal fusion, with improvement of pain and reduction of the disability rate.

IV. The best results in post-operative neurological evolution are shown to be $80 \%$ improvement, with a fusion of $(70 \%)$ and a low rate of pseudoarthrosis in $6(37 \%)$ cases, with patients with kyphosis and decomposed kyphoscoliosis optimally corrected by vertebrectomy, wedge resection of vertebrae and Smith-Peterson osteotomy.

\section{Acknowledgments}

None.

\section{Conflicts of interest}

The author declares there is no conflicts of interest.

\section{References}

1. Moe J. Congenital Deformities of the Spine. In: Bradford D, et al., editors. Spinal deformities, $3^{\text {rd }}$ ed. Madrid: Salvat. 1984;1:135-207.

2. García L. Basic Concepts of Vertebral Surgery. $1^{\text {st }}$ ed. Madrid: Medical Panamericana. 2009;1:21-71.

3. Gomez D, Monterrubio A, Rosales E. Congenital Scoliosis. 49 cases reported. Rev Hosp Jua Mex. 2010;77(2):146-152.

4. Winter R. Congenital scoliosis. Orthop Clin North Am. 1988;19(2):395408.

5. Launay F, Sponseller P. Congenital Scoliosis. In: Herkowitz H, et al., editors. The column, Vol 1, $5^{\text {th }}$ ed. Madrid: Elsevier. 2007;18:507-514.

6. Faundez A, Byrne F, Sylvestre C, et al. Osteotomía de sustracción pedicular en la columna vertebral y toracolumbar, unión torácica: una serie retrospectiva de 28 casos. Diario de Columna de Europa. $2015 ;(24): 42-48$.

7. Gonzalo A, Chanin A, Chamorro M. Ressecao de hemivertebrae com a técnica of eggshel in congenital kyphoscoliosis: results in children with age between one and two years old to seven years. 2009;8(3):337-343.

8. McMaster MJ, Singh H. Natural history of congenital kyphosis and kyphoscoliosis: a study of one hundred and twelve patients. $J$ Bone Joint Surg Am. 1999;81(10):1367-1383.

9. Lenke LG, Sides BA, Koester LA, et al. Vertebral column resection for the treatment of severe spinal deformity. Clin Orthop Relate Res. 2010;468(3):687-699.

10. Gavaskar AS, Chowdary TN. Pedicle subtraction osteotomy for rigid kyphosis of the dorsolumbar spine. Arch Orthop Traum Surg. 2011;131:803-808.

11. R Stelling F, Steel H. Progression of congenital scoliosis due to hemivertebrae and hemivertebrae with bars. J Bone Joint Surg. 1975;57(4):456-466.

12. Gonzalo A, Chanin A, Chamorro M. Pantoja S. Ressecao de hemivertebrae com a técnica de eggshel em cifoescoliosis congénita: resultados em criancas com idade entre um a sete anos. 2009;8(3):337343.

13. Goldberg C, Moore D, Fogarty E, et al. Long-term results from in situ fusion for congenital vertebral deformity. Spine. 2002;27(6):619-628.

14. Suk S, Chung E, Kim W, et al. Posterior vertebral column resection for severe rigid scoliosis. Spine. 2005;30(14):682-687.

15. Villarejo F, Sánchez V. Cirugía de la Columna Vertebral. 1st edition. Madrid Ergon. 2009.

16. Booth KC, Birdwell KH, Lenke LG, et al. Complications and predictive factors for the successful treatment of flat back deformity (fixed sagittal imbalance). Spine. 1999;24(16):1712-1720.

17. Lenke LG, O'Leary PT, Bridwell KH, et al. Posterior vertebral column resection for severe pediatric deformity: minimum two-year follow-up of thirty-five consecutive patients. Spine. 2009;34(20):2213-2221.

18. Lafage V, Blondel B, Smith J, et al. The preoperative planning for osteotomy of pedicle subtraction: Does the inclination pelvic matter? Spine Deform. 2014;2(5):358-366.

19. Jackson RP. Mc Manus AC. Radiographic analysis of sagittal plane alignment and balance in standing volunteers and patient with low back pain matched for age, sex and size a prospective controlled clinical study. Spine. 1994;19(14):1611-1618.

20. Vaz G, Roussouly P, Berthonnaud E, et al. Sagittal morphology and equilibrium of pelvis and spine. Eur Spine J. 2004;11(1):80-87.

21. Leveque J, Edwards A, Rajiv K. After subtraction osteotomy pedicle: An analysis of the parameters radiographic and surgical strategy spine deformity. Spine. 2016;4(3):245-250.

22. Izumi Y, Kumano K. Analysis of sagittal lumbar alignment before and after posterior instrumentation: risk factor for adjacent unfused segment. Eur J Orthop Surg Traum. 2001;1:9-13.

23. Jackson RP, Kanemura T, Kawakami N, et al. Lumbopelvic lordosis and pelvic balance on repeated standing lateral radiographs of adult volunteers and untreated patients with constant low back pain. Spine. 2000;25(5):575-586.

24. Bradford D, Serena S. Empty Transpedicular of the vertebral body. Bradford D. Column. Madrid: Marban. 1999;1:200-208.

25. Dickson R, Leatherman K. Two stage corrective surgery for congenital deformities of the spine. J Bone Joint Surg Br. 1979; 61B(3):324-328

26. Winter RB, Moe JH, Wang JF. Congenital kyphosis: its natural history and treatment as observed in a study of one hundred and thirty patients. J Bone Joint Surg Am. 1973;55(2):223-274.

27. Challier V, Oren J, Bassel D. Eje sagital global: un paso hacia la evaluación de todo el cuerpo de la deformidad plano sagital en el cuerpo humano. J Neurosurg Spine. 2016;15:82-91.

28. Steven DG, Bridwell K, Dimar JR, et al. The impact of positive sagittal balance in adult spinal deformity. Spine. 2005;30(18):2024-2029.

29. Lewis SJ, Gray R, David K, et al. Technique of reverse Smith Petersen osteotomy (SPO) in a patient with fixed lumbar hyperlordosis and negative sagittal imbalance. Spine. 2010;35(15):E721-E725.

30. Lee SH, Kim KT, Suk KS, et al. Sagittal decompensation after corrective osteotomy for lumbar degenerative kyphosis: classification and risk factors. Spine. 2011;36(8):E538-E544.

31. Rose P, Bridwell K, Lenke L, et al. Role of pelvic incidence, thoracic kyphosis, and patient factors on sagittal plane correction following pedicle subtraction osteotomy. Spine. 2009;34(8):785-791.

32. Prahinski J, Polly D, McHale K, et al. Occult intraspinal anomalies in congenital scoliosis. J Pediatr Orthop. 2000;20(1):59-63. 
33. Schwab F, Lafage V, Farcy J, et al. Prediction outcome and complications in the surgical treatment of adult scoliosis. Spine. 2008;33(20):2243-2247.

34. Mac-Thiong J, Transfeldt E, Mehbod A, et al. Can C7 plumb line and gravity line predict health related quality of life in adult scoliosis? Spine. 2009;34(15):E519-E527.

35. Schwab F, Lafage V, Patel A, et al. Sagittal plane considerations and the pelvis in the adult patient. Spine. 2009;34(17):1828-1833.

36. Boulay C, Tardieu C, Hecquet J, et al. Sagittal alignment of spine and pelvis regulated by pelvic incidence: standard values and prediction of lordosis. Eur Spine J. 2006;15(4):415-422.
37. Legaye J, Duval-Beaupère G, Hecquet $\mathrm{J}$, et al. Pelvic incidence: a fundamental pelvic parameter for three-dimensional regulation of spinal sagittal curves. Eur Spine J. 1998;7(2):99-103.

38. Pedroza A, Tavares R, Limoiera A. Posterior vertebrectomy for the treatment of spinal congenital deformities Coluna/Columna. Spine. 2007;6(3):168-1673.

39. Deviren V. In correspondence. J Bone Joint Surg Br. 2002;84B(3):305308. 